\title{
El clima urbano de ciudades subtropicales costeras atlánticas: el caso de la conurbación de Florianópolis ${ }^{1}$
}

\author{
Magaly Mendonça² y Magda A. Lombardo ${ }^{3}$
}

\begin{abstract}
RESUMEN
Este estudio del clima de la conurbación de Florianópolis demuestra la influencia simultánea de los factores geoecológicos y de la urbanización sobre la variación espacio-temporal de la temperatura y otros elementos climáticos. Se levantaron datos meteorológicos en varias localidades, en horarios sinópticos y en diferentes tipos de tiempo, utilizando un termo hidrógrafo digital y observaciones visuales. Los mapas de gradiente de temperatura, referidos a datos de la estación del aeropuerto, demostraron una significativa conservación térmica en los locales ocupados por construcciones verticales, de alta densidad e intenso tráfico. No se observó la formación de una isla de calor continua, sino que un archipiélago, debido tanto al carácter multinuclear de la urbanización como a la compartimentalización morfológica de los sitios. Las islas de calor más intensas se registraron en el sector central de la región, bajo dominio polar y en situaciones de transición y prefrontal. Estos resultados pueden ser importantes para la evaluación de la calidad ambiental, formando parte de los diagnósticos necesarios para el planeamiento urbano.
\end{abstract}

Palabras clave: Islas de calor, tipos de tiempo, uso del suelo.

\begin{abstract}
This work demonstrates the simultaneous influence of geoecological and urban factors on the variation of temperature and other climatic elements of the Florianópolis conurbation. Data was obtained in various places, following synoptic time series, under different weather types, using a digital thermo hygrometer and visual information. The temperature gradients maps, compared with airport station, demonstrated a significant thermal conservation capacity in places occupied by buildings and dense and intense traffic. Urban heat archipelagos instead of a continuing urban heat island were recorded as a consequence of urban poly nucleus and site morphologic compartments. The most intense heat islands occurred in the central part of the region, when the polar air masses dominate or are under pre-frontal transition. These results are relevant for the evaluation of environmental quality, and should support urban planning diagnostics.
\end{abstract}

Key words: Urban heat islands, weather types, land use.

\footnotetext{
1 Artículo recibido el 10 de junio de 2008 y aceptado el 7 de mayo de 2009.

2 Departamento de Geociencias, Universidad Federal de Santa Catarina (Brasil).

E-mail:magaly@cfh.ufsc.br
}

\footnotetext{
Departamento de Cartografía y Análisis de Información Geográfica, Universidad Estadual de São Paulo (Brasil).E-mail: lombardo@rc.unesp.br
} 
Este estudio se relaciona con los condicionantes geoecológicos y ambientales que definen el clima de la región conurbada de Florianópolis, buscando un mejor entendimiento de los mecanismos y procesos determinantes del clima regional, de las características y ritmos del clima local, de las modificaciones impuestas por los atributos urbanos y de las consecuencias de estas interferencias en las variaciones de las temperaturas y demás elementos meteorológicos en los espacios de vivencia cotidiana.

El área estudiada se localiza en el estado de Santa Catarina, ubicado en el sudeste de Brasil, 705 km al sur de São Paulo, ciudad capital, y entre los estados de Paraná (ciudad capital Curitiba) y Río Grande do Sul (ciudad capital Porto Alegre). Este estudio comprende la ciudad de Florianópolis y la parte central de la costa catarinense, además de São José y parte de los municipios de Palhoça y Biguaçu (entre $27^{\circ} 23^{\prime} \mathrm{S}$ a $27^{\circ} 55^{\prime} \mathrm{S}$ y $48^{\circ} 20^{\prime} \mathrm{W}$ a $\left.48^{\circ} 45^{\prime} \mathrm{W}\right)$. La mayor parte de la región es una isla, y Florianópolis, la capital, forma una conurbación con los demás municipios, concentrando una población de 651.098 habitantes, correspondientes al $14,9 \%$ de la población urbana estadual (IBGE, 2000). Florianópolis ejerce el papel de polo político-administrativo-financiero, centralizando gran parte de los servicios de asistencia médica, educacionales y culturales, utilizados por la mayor parte de la población del estado (Mendonça, 2002). Durante los últimos años se ha constituido en un importante destino para el turismo nacional e internacional, ocupando el primer lugar en las preferencias de los chilenos en el verano de 2008.

El análisis de la situación actual del clima de la ciudad y el establecimiento de directrices de planeamiento son fundamentales para minimizar los efectos negativos de la urbanización sobre el medio ambiente y la calidad de vida de la población. Esta es una cuestión que está en la pauta de las preocupaciones globales, tanto en función del constatado aumento de los gases de efecto invernadero, principalmente del dióxido de carbono que se concentra especialmente sobre la atmósfera de las ciudades, como debido a la importancia de los cambios climáticos sobre la salud y bienestar de la población urbana de Brasil, que equivale al $90 \%$ de la población nacional. El modelo económico urbano-industrial, seguido por este país, en su fase monopolista, ha promovido la concentración de la población y de los recursos y ha acentuado y propagado los problemas urbanos.

\section{El paisaje en la región}

La costa catarinense está formada por secuencias de sierras dispuestas de forma subparalela, en sentido norte-sur, con altimetría baja en dirección al litoral, terminando en puntas, penínsulas e islas (Rosa y Herrmann, 1986). En el municipio de Palhoça se registran las mayores elevaciones, con ápice en 915 m de altitud. En São José y Biguaçu las mayores alturas alcanzan entre 490 m y 540 m. En el sur de la isla de Santa Catarina el punto culminante alcanza 532 $\mathrm{m}$. Las alturas medias de la región estudiada son inferiores a $300 \mathrm{~m}$.

Entre las elevaciones rocosas existen planicies costeras originadas por procesos marinos, aluviales y coluvio-aluvionales. La planicie marina de forma plana alcanza altitudes de hasta $5 \mathrm{~m}$, por la presencia de remanentes de cordones de playa y terrazas marinas. En la planicie aluvial las terrazas presentan altitudes entre $5 \mathrm{~m}$ y $10 \mathrm{~m}$, sin constituir un contacto abrupto. Las rampas coluvio-aluvionales son formadas por superficies descontinuadas, poco inclinadas, con alturas en torno de $10 \mathrm{~m}$ a $20 \mathrm{~m}$, constituidas por depósitos en los soportes de las vertientes y aluviones subactuales (Herrmann, 1999).

La combinación entre sierras y planicies dio origen a un litoral recortado, con innumerables playas, puntas, promontorios, islas y lagunas. Sobre las planicies, en la costa este, donde los vientos dominantes son más intensos y canalizados, ocurren dunas, dispuestas en lençóis, en grandes corredores.

Entre las porciones entre la isla y el continente se localizan amplias bahías al norte y al sur, separadas por un estrecho canal de $500 \mathrm{~m}$ de ancho, sobre el cual los puentes permiten la conexión entre la isla y el continente. Al interior de esas bahías desaguan ríos cuyos bordes presentan vegetación de 
manglares. Sobre las laderas de la sierra costera se encuentran aún remanentes de la selva Ombrófila densa (mata atlántica), en los puntos de alta inclinación. En muchos cerros de la región la vegetación secundaria, en avanzado estado de regeneración, ocupa espacios antes dedicados a la agricultura. Las planicies cubiertas por formaciones pioneras (vegetación de playa, dunas y manglares) fueron por mucho tiempo utilizadas para prácticas agrícolas que en las últimas décadas han estado siendo sustituidas por la ocupación urbana, reforestación con pinos, cultivos de arroz, pastizales o vegetación nativa en recuperación (Governo do Estado de Santa Catarina, 1996). Parte de la vegetación nativa está protegida por la constitución de Unidades de Conservación Ambiental, principalmente en la isla y en el municipio de Palhoça. Sin embargo, tales áreas también han estado siendo sometidas clandestinamente a actividades inadecuadas.

Hasta la década de 1970 el proceso de urbanización de la región se caracterizaba por una estructura de núcleos urbanos con bajas concentraciones poblacionales. En 1960, solamente Florianópolis presentaba una población urbana superior a la rural. A partir de entonces el desarrollo de la red vial, que privilegió el flujo de transporte entre los centros urbanos y la modernización industrial junto a los ejes de las carreteras, favorecieron la concentración urbana y el crecimiento de otras ciudades en municipios como São José, Biguaçu y Palhoça.

En Florianópolis la instalación de órganos gubernamentales estatales y federales y de una extensa red de servicios fueron los verdaderos factores inductores del crecimiento de la población. Este crecimiento se reflejó en una nítida expansión del sector inmobiliario, con el inicio de una acelerada verticalización de la ocupación en la década de 1970 en el centro histórico y la diseminación de funciones centrales para las demás áreas de la región, a partir de la década de 1980. Actualmente esta expansión alcanza a toda la región, principalmente a los balnearios, en función del incremento del apoyo gubernamental a las actividades de turismo.
Herrmann (1999) identificó una serie de problemas relacionados especialmente con el uso inadecuado del suelo y la infraestructura urbana, los que interfieren negativamente en la calidad ambiental y bienestar de la población. Entre aquellos que más interfieren en la configuración del campo térmico, se destacan con algunas adaptaciones las siguientes:

- La impermeabilización del suelo con revestimientos de asfalto y cemento, principalmente en las áreas sedimentarias.

- La ocupación de las costas con loteamientos y edificaciones en áreas de regeneración del bosque, que había sido devastado en épocas anteriores para explotaciones agrícolas.

- La ocupación urbana de áreas de preservación ambiental como manglares, dunas y áreas de protección de los recursos hídricos.

- Implantación de rellenos en áreas de planicies de inundación y ampliación de los rellenos sobre áreas marinas para edificaciones y ampliación del sistema viario.

- Densificación de la ocupación horizontal y vertical de forma espontánea o inducida por la legislación y las políticas urbanas, creando condiciones para la formación de islas de calor.

\section{El clima subtropical húmedo y su expresión regional/local}

La región conurbada de Florianópolis, por su posición subtropical a $27^{\circ} \mathrm{S}$ (Figura $N^{\circ}$ 1) sobre la costa atlántica, presenta características climáticas controladas por el predominio de altas temperaturas, elevada precipitación y contenido de humedad atmosférica, lo que la diferencia netamente de las ciudades litorales áridas de la costa pacífica (Caldera y Copiapó), ubicadas a la misma latitud y situadas en medio del margen austral del desierto de Atacama. Las condiciones subtropicales cálidas controladas por masas de aire tropical marítimo son interrumpidas por la penetración, actuación y choque de las masas Polar Marítimas del Atlántico, originando veranos calientes e inviernos frescos, típicos de los climas subtropicales de la costa oriental de América del Sur. Los días cálidos y húmedos son modifi- 
cados por el paso de frentes fríos polares, que ocasionan bruscos cambios del tiempo atmosférico en cualquier estación del año. Siempre precedida por la elevación de la temperatura, en invierno, el paso de los frentes es sucedido por ondas de frío causadas por las masas polares, con ocurrencia de heladas en las áreas menos urbanizadas. En el verano, las condiciones subtropicales del clima registran la instalación de las masas de aire polar, a través del cambio en la dirección de los vientos, que siendo del noreste, pasan a soplar desde el sur, y por una agradable reducción de las temperaturas después de días de calor intenso.

En función de la actuación de masa de aire Polar Marítima, el tiempo puede presentar una gran amplitud térmica diaria y una humedad inferior a 50\%, aunque normalmente debido a la influencia de la maritimidad la humedad relativa media es alta, en torno al $80 \%$. Los vientos predominantes soplan del cuadrante norte y los más veloces y también frecuentes son del sur, asociados a las masas de aire Tropical Marítima y Polar Marítima del Atlántico, res- pectivamente. Los vientos del sur anteceden la entrada de los frentes fríos y del aire Polar Marítimo con ráfagas de hasta 80 km/h (Monteiro, 1992).

El ritmo de las precipitaciones está regulado también por el Frente Polar, puesto que las Iluvias son generalmente prefrontales, frontales y posfrontales. Sin embargo, son menos abundantes en invierno; leves, continuas y más rápidas y torrenciales en verano, principalmente en febrero. En esta estación ocurren Iluvias convectivas, asociadas al calentamiento del continente, que junto al litoral, presenta temperaturas medias máximas, en febrero, debido al atraso del calentamiento de las aguas en relación al solsticio de verano. Las precipitaciones estivales caracterizan el clima subtropical húmedo de fachadas orientales propuesto por Strahler (1975).

Los sistemas frontales son activos en todas las estaciones del año, avanzan hacia latitudes tropicales e influencian los regímenes de precipitaciones y temperatura de gran parte de Brasil (Climanálise, 1986).

Figura $\mathrm{N}^{0} 1$

Localización del área de estudio

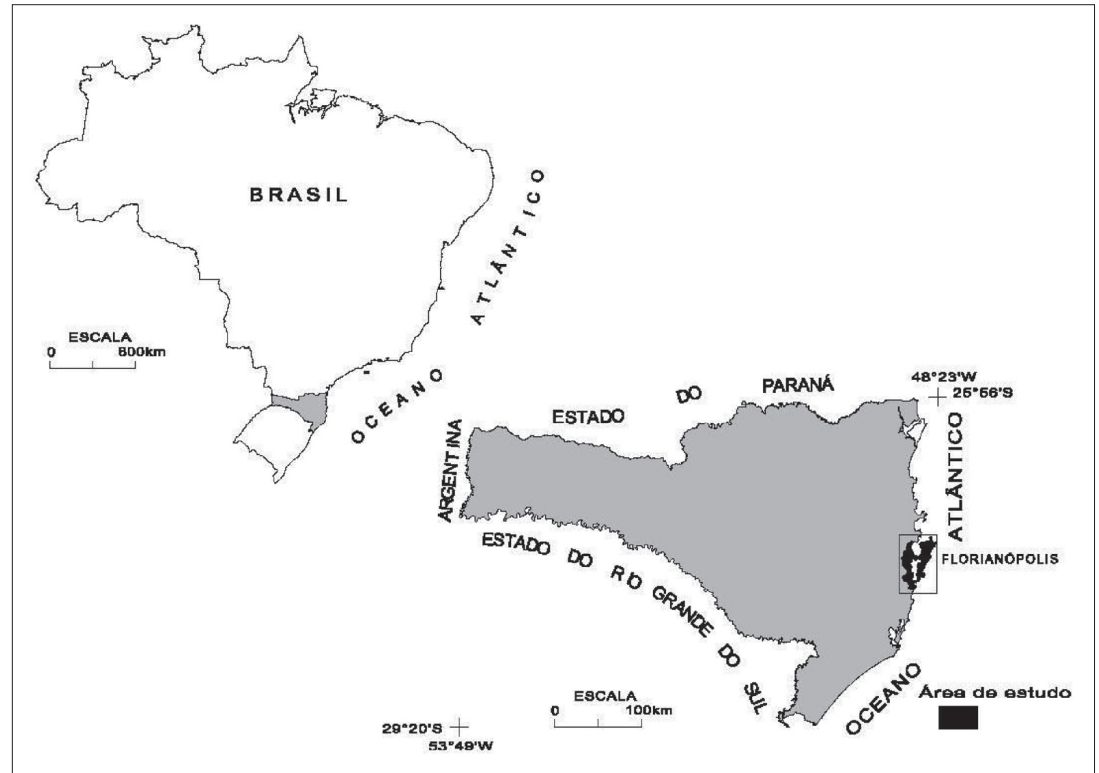

Fuente: Mendonça et al., 2007. 
Monteiro (1963), sobre la base de los aportes de la meteorología dinámica desarrollada en Brasil por Serra (1954) y Serra \& Ratisbonne (1942), propuso una esquematización del mecanismo general promedio del frente polar en una secuencia típica de tiempo que, repitiéndose en el correr del año, forma un verdadero ciclo que con mayor o menor nitidez comprende las siguientes fases o situaciones: transición, prefrontal, avance frontal y dominio polar. Este modelo no era considerado válido para el verano, cuando a lo más se caracterizaría como una onda de frío. Entretanto, el referido autor perfeccionó la clasificación cuando caracterizó los tipos de flujos polares en continuo, dominante, alternado (oscilante), interrumpido, débil y nulo (Monteiro, 1969). La intensidad de los diferentes flujos está asociada a la capacidad de acumulación del aire frío en el sur del continente y consecuentemente también a las estaciones del año.

Debe considerarse que, incluso con el debilitamiento del anticiclón migratorio polar en verano, el mecanismo general de circulación atmosférica continúa siendo desencadenado por el Frente Polar aunque a un ritmo diferente. La caracterización de los flujos polares comporta una secuencia típica de tiempo también en el verano. Monteiro (1963) admite que la secuencia no es siempre tan nítida.

En la presente investigación se consideró importante no limitar el análisis a una estación del año, pero sí a los tipos de tiempo, percibiéndose que dentro de un análisis geográfico siempre es posible caracterizar las condiciones observadas en la región en referencia a estos modelos. El análisis temporo-espacial del período de registro en la región se basó en estos modelos.

\section{Materiales y métodos}

Se realizó un mapa de uso de la tierra utilizando técnicas de percepción remota y geoprocesamiento, mediante el cruce de dos imágenes satelitales, una de SPOT P del 7 de noviembre de 1995, con resolución espacial de $10 \mathrm{~m}$, y otra de LANDSAT ETM+ del 25 de agosto de 1999, coincidentes con el año de los levantamientos meteorológicos. La clasificación supervisada fue desarrollada a través del programa Idrisi for Windows versión 2.

Para Monteiro (1990b), los valores meteorológicos registrados por un punto resultan de la combinación de los componentes de la circulación atmosférica regional con los atributos locales, reflejando al mismo tiempo el contexto mesoclimático definido por las grandes líneas de la topografía y principalmente las condiciones microclimáticas creadas por la edificación urbana. Esta ponderación fue la que orientó la proposición de un zoneamiento mesoclimático, la clasificación de los usos e incluso el análisis de los datos levantados.

Para la selección preliminar de las áreas para establecimiento de puntos de observación meteorológica fueron considerados como criterios la compartimentación morfológica, altitud y uso de la tierra, así como áreas con diferentes grados de urbanización. Con base en el material cartográfico y reconocimiento en el campo, fueron seleccionadas treinta áreas próximas a las residencias o lugar de trabajo de los auxiliares para los levantamientos. Las áreas escogidas fueron distribuidas por toda la isla y el continente, en la medida de lo posible, y en los municipios de São José, Biguaçu y Palhoça. Fueron montadas estaciones en el norte y sur de la región y concentradas en el sector central de la misma, que es el más urbanizado.

Para la realización de los registros meteorológicos se procuró establecer estaciones de sondaje en lugares con concentraciones de edificios de diferentes alturas; pastos, bosques, suelos desnudos, edificaciones claras u oscuras (albedo); parques para estacionamiento de vehículos según su pavimentación, edificaciones no planeadas (espontánea, informal, clandestina), favelas, y distintos materiales de construcción, coloración y densidad. Se buscó, inclusive en una misma localidad, la existencia de patrones diferentes de densidades de construcciones y usos, para el establecimiento de dos estaciones, cuya distancia entre ellas permitiese que los registros fuesen realizados en aproximadamente 20 minutos.

Para seleccionar los instrumentos para el trabajo de campo fueron consideradas la na- 
turaleza de los levantamientos y las necesidades de transporte de los mismos y de los auxiliares de los registros. Esto exigía liviandad, fácil manipulación y resistencia a los derribamientos por el viento. Además de eso, adecuación a una investigación científica, en términos de calidad, precisión y costo. Sobre la base de estos criterios se escogió el termohigrómetro digital TFA, producido en Alemania, que registra la temperatura en grados Celsius entre $-10^{\circ} \mathrm{C}$ y $+50^{\circ} \mathrm{C}$ y la humedad entre $10 \%$ y $99 \%$. Fue necesario también concebir un aparato liviano para sustentarlo a 1,5 m encima del suelo y la elaboración de material de apoyo para los auxiliares de los registros en el campo: instrucciones detalladas, fichas para anotaciones, etc.

La precisión de los termohigrómetros fue probada con un conjunto de 30 unidades en el mismo ambiente y con otros modelos. Cinco unidades fueron probadas en la estación del Aeropuerto, durante 24 horas, que fueron consideradas adecuadas para levantamientos meteorológicos.

Para Monteiro (1990a), ante la normal dificultad de disponer de recursos para la adquisición de instrumentos sofisticados, los aparatos simples distribuidos por la ciudad pueden proveer informaciones, aunque discretas y singulares, sobre las relaciones de los hechos termodinámicos básicos del aire como respecto a los rasgos específicos de la propia condición urbana. La simplicidad de los aparatajes debe ser compensada por la homogeneidad de los instrumentos de observación y la multiplicación de los puntos de registro en el universo de análisis. Es imprescindible que el instrumental utilizado, como el tipo de abrigo y la distancia del suelo, sean multiplicados en condiciones idénticas por todos los puntos (Monteiro, 1990b).

Los métodos de levantamiento, inspirados en Monteiro y Sezerino (1990), procuraron estar mínimamente en sintonía con las normas establecidas por la Organización Meteorológica Mundial para observaciones de superficie, buscando la obtención de datos comparables con aquellos registrados en estaciones oficiales. A pesar de que el objetivo de captar el campo térmico urbano compromete las normas de localización, se procuró establecer un distanciamiento mínimo de las edificaciones, tomar la temperatura a 1,5 $\mathrm{m}$ de la superficie y obedecer a los horarios sinópticos, inclusive en los meses en que fue instituido el horario de verano.

Los levantamientos fueron realizados en los meses de abril, julio y octubre de 1999 y febrero de 2000, durante 4 días, de viernes a lunes, para evaluar la influencia de la funcionalidad urbana y la estructura (sitio, espacio construido) de la ciudad. Los levantamientos fueron hechos a las 6 hrs., 9 hrs., 12 hrs., 15 hrs., 18 hrs. y 21 hrs., relativos a los horarios sinópticos $(9,12,15,18,21$ y 24 GMT). Los horarios de las 9 hrs., 15 hrs. y $21 \mathrm{hrs}$. fueron seleccionados para coincidir con aquellos registrados en la estación meteorológica principal, y los horarios de las 6 hrs. y 18 hrs. para evaluar también la conservación del calor en ausencia de la radiación solar. Los horarios de las 6 hrs. y 21 hrs. son frecuentemente referidos en la bibliografía para evaluación del comportamiento de las "islas de calor".

Después de cada uno de los levantamientos de campo fueron construidas tablas con los datos levantados: temperatura del aire, humedad relativa del aire, tráfico por minuto, luz/sombra sobre la estación, dirección del viento, intensidad del viento a partir de la Escala Beaufort, nubosidad, visibilidad, tipos de nubes y ocurrencia o no de Iluvia, con criterios para su calificación.

Para la sistematización y análisis de los datos de todas las estaciones, estos fueron digitados en planilla electrónica buscando la organización de tablas y gráficos. Además, desde el año 1999 a febrero del 2000 fue hecha una búsqueda sistemática, a través de Internet, en páginas de instituciones que abordan temas de meteorología y climatología respecto a la recolección de cartas sinópticas, imágenes de satélites y análisis del tiempo. Esta búsqueda tuvo por objetivo caracterizar el clima regional y local, además de clasificar el cuadro sinóptico del período en que fueron hechos los levantamientos de campo.

Para Monteiro (1990b), además de los datos meteorológicos de la estación patrón en el mismo período de los levantamientos, 
importa también la variación diaria mensual en las ciudades en estudio, posibilitando la validación "de las 'constantes' debidas a los sistemas meteorológicos regionales y las 'variables' impuestas por las características 'locales'" (Monteiro, 1990b: 63).

En el análisis de las condiciones del tiempo y circulación regional fueron hechas comparaciones de los datos meteorológicos de Florianópolis con aquellos de Porto Alegre y Curitiba, a través de la página del Instituto Nacional de Meteorología (INMET) que presenta las condiciones meteorológicas registradas en las capitales regionales brasileñas. Las estaciones de observación del patrón son imprescindibles en la comparación de las observaciones complementarias.

La cuestión de las escalas ha sido tratada por muchos autores, manteniéndose hasta hoy como una cuestión controversial, principalmente en términos del tamaño espacial. Este trabajo no tuvo por objetivo discutir la cuestión de las escalas, sino que abordar un clima local y urbano, a través de la articulación de las escalas espaciales y temporales. Para entender la distribución de la temperatura y la humedad relativa del aire en la región conurbada de Florianópolis, se consideró indispensable, además de reconocer los tipos de tiempo, identificar también la situación de macroescala y jerarquizar las manifestaciones climáticas en los demás niveles durante el período de levantamientos de campo. Así se buscó caracterizar desde el clima zonal hasta los microclimas levantados durante los registros episódicos, a partir de una secuencia de tipos de tiempos ya definida.

Para la representación del campo térmico fueron producidos mapas de isotermas para cada uno de los episodios de levantamiento de los datos meteorológicos: abril, julio, octubre, noviembre y febrero. Para cada día de levantamiento fueron hechos mapas para los horarios de las 6 hrs., 9 hrs., 15 hrs. y 21 hrs., con el objetivo de mostrar las mayores diferencias de temperatura y la conservación térmica que configura las islas de calor.

Los mapas fueron luego sometidos a un análisis visual, apoyado en los datos tabulados. Como tal análisis resultó insuficiente, fue necesaria una profundización de las in- vestigaciones. Como ello no posibilitó conclusiones inequívocas, se procuro combinar los datos de cuatro días de cada levantamiento y a partir de ahí buscar alguna posibilidad de clasificación de los resultados.

Inicialmente se buscó clasificar los lugares de las estaciones a través del examen de los datos de cada horario y de cada día, utilizándose como referencia regional la estación meteorológica del Aeropuerto.

La estación meteorológica del Aeropuerto fue escogida al constatarse que la mayor parte de sus registros presentó temperaturas más bajas que aquellas registradas en la estación climatológica principal de Florianópolis, de la red del INMET y también porque los registros son realizados a cada hora. Esto permitió la comparación en todos los horarios seleccionados para los levantamientos, principalmente por el registro en el horario de las 6 hrs., considerado como representativo de la temperatura mínima diaria. En la estación del INMET los registros existen solo a las 9 hrs., 15 hrs. y 21 hrs.

Después de la determinación de la estación del Aeropuerto como referencia, los datos de temperatura del aire de un levantamiento dado (por ejemplo del 16/04/99 al 19/04/99) fueron organizados por horario en tablas, en una planilla electrónica Quattro Pro. Las estaciones fueron dispuestas una abajo de la otra, con los respectivos datos de temperatura del aire al lado, en otra columna. Así fue posible extraer el delta de la temperatura de cada estación, estableciendo una clasificación creciente y decreciente en relación a la estación de referencia.

Con el delta fueron confeccionados mapas con los gradientes de temperatura que posibilitaran una mejor visualización de las islas de calor y frescor.

\section{Análisis espacio-temporal de las relaciones en la dinámica paisajística}

Las diferencias de temperatura registradas a las 9 hrs., 15 hrs. y 21 hrs., en muchas estaciones, denotan la influencia del sitio, puesto que el proceso de calentamiento-en- 
friamiento estuvo directamente ligado a la exposición del plano de inclinación de la pendiente en relación al movimiento del sol (Mendonça y Lombardo, 2002).

Algunas estaciones no representativas del clima urbano, que presentaron conservación térmica, sufren influencias de otros factores de escala topo a microclimática, que merecen mayor investigación, especialmente ante la perspectiva de una densificación en la ocupación.

La distribución de las temperaturas registradas es característica de los fenómenos "isla de calor" e "isla de frescor", con nítidos contrastes térmicos entre los espacios con ocupación urbana acentuada y otros que presentan diferentes grados de urbanización.

Los datos levantados demostraron que las edificaciones verticales, principalmente donde son densas, como ocurre en el centro de Florianópolis, funcionan como eficientes almacenadores térmicos. Durante la tarde el sombreamiento causado por la verticalización reduce las diferencias registradas en esa área. Pero las temperaturas registradas a las $21 \mathrm{hrs}$. y a las $6 \mathrm{hrs}$. del día siguiente atestiguan la conservación térmica.

Las mayores diferencias de temperatura coinciden, en el mapa de uso de la tierra, con las áreas clasificadas como urbanas densas, localizadas principalmente en las zonas Sede y São José, y que corresponden a $0,78 \%$ del total del área estudiada. Diferencias en torno a $\operatorname{los} 2,0^{\circ} \mathrm{C}$ coinciden con las áreas urbanas menos densas (3,08\%), que se localizan en núcleos o a lo largo de las rodovías en toda la región.

Quedó demostrado también que, para la temperatura tomada a 1,5 $\mathrm{m}$ del suelo, la altitud, la presencia de cobertura vegetal densa, condiciones favorables a la ventilación y la proximidad de cuerpos de agua (lagunas o mar) minimizan el efecto de los atributos urbanos.

Registros realizados en estaciones próximas, pero con densidades distintas, revelaron diferencias en torno de $1,0^{\circ} \mathrm{C}$ y hasta de $1,5^{\circ} \mathrm{C}$ entre ellos.
En las investigaciones episódicas se procura captar las diferentes situaciones de tiempo, buscando explicitar el comportamiento de la temperatura en la región conurbada de Florianópolis frente a las variaciones climáticas estacionales, como también en los diferentes días de la semana, desde el punto de vista de la funcionalidad, en una visión procesal y dinámica.

Caracterizando las islas de calor más intensas, las mayores diferencias de temperatura fueron observadas bajo el dominio polar a las 6 hrs., horario en que normalmente ocurren las mínimas (Figura $\mathrm{N}^{\circ} 2$ ). En el levantamiento episódico de abril, bajo el dominio del flujo polar dominante, fueron registradas diferencias superiores a $7,0^{\circ} \mathrm{C}$. Bajo el dominio del flujo polar alternado, el 22 de julio fueron observadas diferencias superiores a $3,0^{\circ} \mathrm{C}$, pero en octubre las diferencias alcanzaron los $9,0^{\circ} \mathrm{C}$ en el centro de Florianópolis.

En abril, se notó que la diferencia de temperatura registrada a las 6 hrs. fue disminuyendo del primer al tercer día de dominio polar, mientras que aquellas registradas a las $15 \mathrm{hrs}$. y $21 \mathrm{hrs}$. fueron aumentando, alcanzando valores superiores a $5^{\circ} \mathrm{C}$ el día 19

A fines de noviembre, aún en primavera, pero bajo condiciones típicas de tiempo de verano, las mayores diferencias, superiores a $5^{\circ} \mathrm{C}$, fueron registradas a las $15 \mathrm{hrs}$., en el dominio de un flujo polar nulo. En febrero la misma situación fue observada en tipos de tiempo como el de transición, prefrontal y avance. Tanto en noviembre como en febrero, a las 6 hrs., las diferencias fueron superiores a $3,0^{\circ} \mathrm{C}$, excepto en la situación de prefrontal cuando sobrepasaron $\operatorname{los} 6,0^{\circ} \mathrm{C}$, inclusive a las 15 hrs. (Figura $N^{\circ} 3$ ). Esta es una situación más crítica en términos de calidad ambiental porque elevadas temperaturas se combinan con la elevada humedad relativa del aire, causando el aumento de la sensación de calor.

Las islas de frescor fueron registradas en todos los tipos de tiempo y horarios, pero siempre en las estaciones situadas en lugares más abiertos, próximos al mar, lagunas, vegetación de manglares, en altitudes más elevadas o en amplias planicies. 
En abril, bajo condiciones de dominio de flujo polar fuerte, con cielo despejado y calmas, las islas de frescor ocurrieron a las 6 hrs. y 21 hrs., en las localidades menos urbanizadas. A las $15 \mathrm{hrs}$. su registro se asoció con brisas más fuertes (cinco a seis grados en la Escala Beaufort) y cielo despejado, en los balnearios. Bajo el dominio de flujos polares más débiles, ocurrieron en condiciones de brisas también más débiles y mayor y/o cobertura del cielo, en general, inversamente proporcional a la intensidad de las brisas. En esas condiciones cubrieron espacios más amplios a las 6 hrs. el día 5 de julio y a las 21 hrs. el 18 de octubre.

En el centro de Florianópolis no fueron detectadas islas de frescor a las 6 hrs., como tampoco en las proximidades de la plaza próxima a la Catedral. Solo a las $15 \mathrm{hrs}$. del día 5 de julio se verificó la configuración de una isla de frescor que se extendió hasta un barrio próximo del centro. Esta se opone a una pequeña isla de calor, con temperatura en torno a $\operatorname{los} 2,0^{\circ} \mathrm{C}$ superiores a la registrada en el Aeropuerto, alcanzando a las estaciones del centro.

La influencia de la funcionalidad puede ser mejor observada los días 18 (domingo) y 19 (lunes) de abril, pues ambos reflejaron condiciones de dominio polar. En la comparación entre los mapas de esos días se percibe que, cuando fueron intensificadas las actividades urbanas (lunes), ocurrió una ampliación de la extensión de las islas de

Figura $\mathrm{N}^{\circ} 2$

Mapas del gradiente de temperatura en abril de 1999

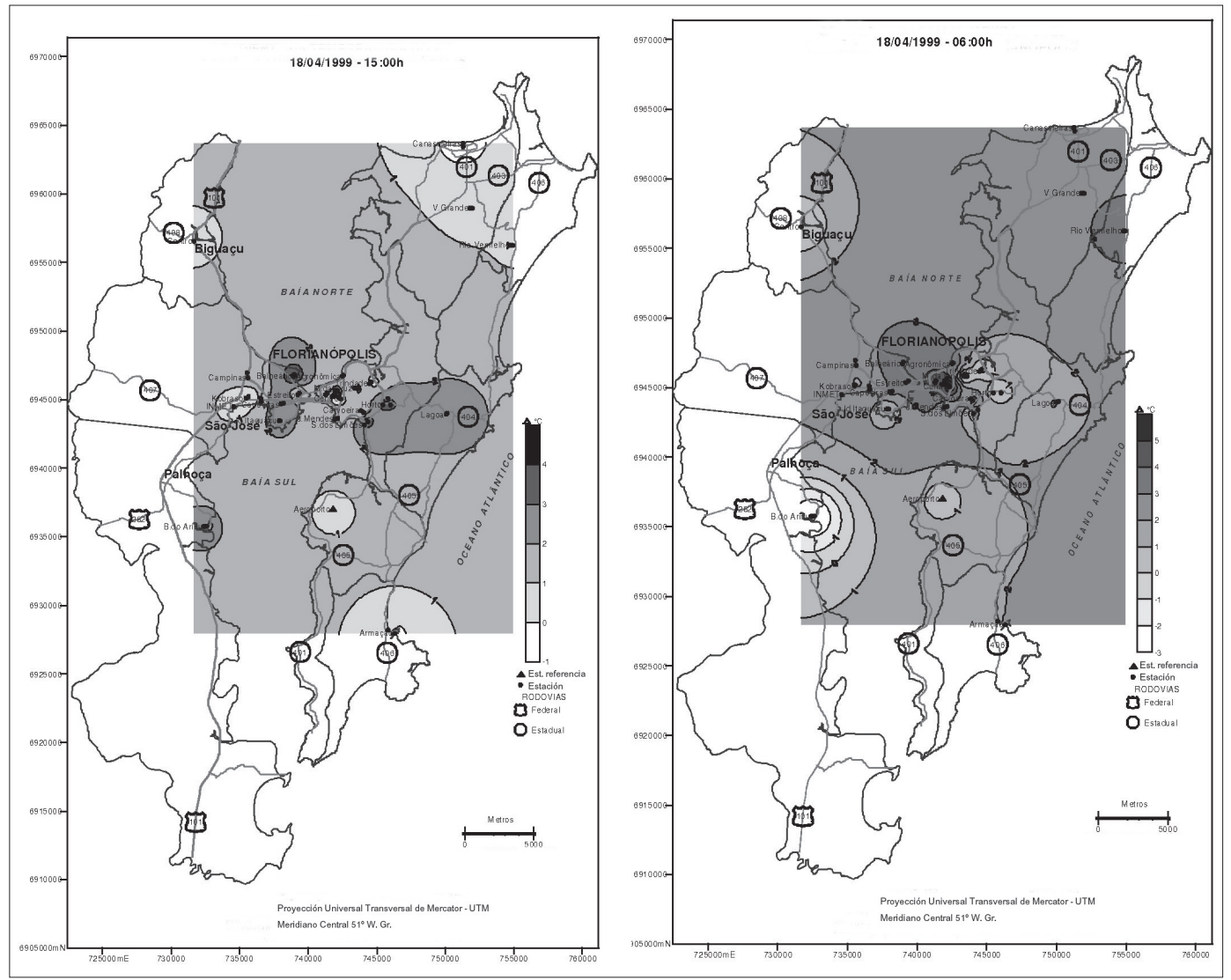

Fuente: Mendonça, 2002. 
calor y un aumento de las diferencias de temperatura en las estaciones comprendidas por ella.

La humedad relativa del aire, en todos los levantamientos episódicos, estuvo sujeta a las variaciones de la temperatura, independientemente de la cantidad de vapor. En la mayoría de las veces el comportamiento de la humedad siguió de cerca el de la temperatura, coincidiendo la presencia de islas de calor con islas secas. Incluso en los lugares donde la urbanización fue más efectiva la humedad relativa nocturna fue mayor que la diurna. El comportamiento de la humedad relativa del aire estuvo siempre asociado a la circulación atmosférica, siendo ella más elevada en el prefrontal y avance y sensible- mente más baja en la fase inicial de la instalación del dominio polar.

En la región conurbada de Florianópolis las islas de calor se manifestaron persistentemente en el sector central, comprendiendo las estaciones del este del morro de la Cruz, el centro y continente de Florianópolis y São José. Ellas ocurrieron en todos los tipos de tiempo relativamente estables, excepto el cuatro de julio a las $15 \mathrm{hrs}$. y el 15 de octubre a las 21 hrs., variando en áreas de extensión, alcance e intensidad; en general, fueron más amplias e intensas a las 21 hrs.; aunque en la instalación e inicio del dominio polar las mayores diferencias hayan sido registradas a las 6 hrs. y en la transición y prefrontal a las $15 \mathrm{hrs}$.

Figura $\mathrm{N}^{\circ} 3$

Mapas del gradiente de temperatura en febrero de 2000

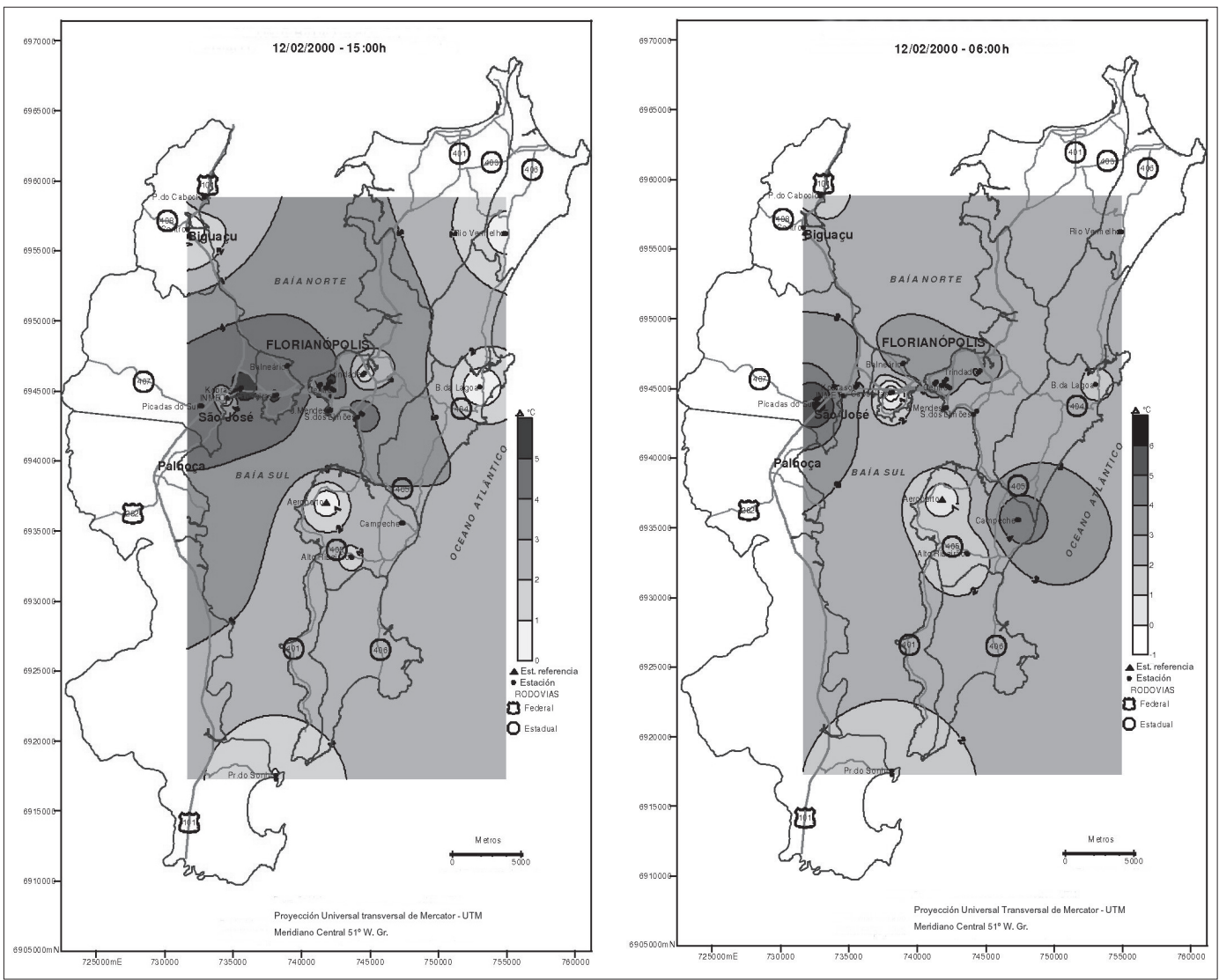

Fuente: Mendonça, 2002. 
Debido al carácter multinuclear de la urbanización y de la compartimentalización morfológica del sitio, no se verificó el modelo clásico de la isla de calor en forma de domo, con disposición concéntrica de las isotermas, ni una configuración de una única isla. Lo que se configuró fue un "archipiélago", como habían previsto Sezerino y Monteiro (1990).

Los contrastes de temperatura verificados en este archipiélago sobrepasaron, tanto en situaciones de dominio polar como prefrontal, las variaciones señaladas por la literatura, que serían de apenas $5,0^{\circ} \mathrm{C}$ de temperatura entre la ciudad y el campo en latitudes extratropicales. En São Paulo, Lombardo (1985), y en Porto Alegre, Danni (1987), ya habían detectado situaciones semejantes. En São Paulo este fenómeno ocurre de forma intensa, habiendo sido verificado un gradiente de temperatura horizontal superior a $10^{\circ} \mathrm{C}$, el mayor constatado en un tipo de tiempo estable y con calma. Para Lombardo (1985), esta anomalía refleja no solo la gran dimensión de la mancha urbanizada, sino que también la distribución de los diferentes tipos de uso de la tierra que asociados a situaciones sinópticas imprimen un dinamismo tanto a niveles diario y semanal como estacional.

En la región conurbada estas anomalías se manifestaron frecuentemente en torno de los $6,0^{\circ} \mathrm{C}$, pero en situaciones de dominio polar, a las 6 hrs., en estaciones localizadas en el centro de Florianópolis, sobrepasaron $\operatorname{los} 7,0^{\circ} \mathrm{C}$ y $\operatorname{los} 9,0^{\circ} \mathrm{C}$. Gómez y García (1984) verificaron para Madrid diferencias medias en torno de los $4^{\circ} \mathrm{C}$ y $7^{\circ} \mathrm{C}$ durante el invierno en las mínimas absolutas en relación a la referencia. La situación verificada por ellos se asemeja significativamente a la observada en la región estudiada.

\section{Consideraciones finales}

El gran desafío metodológico de este trabajo fue analizar el clima local, en la escala de la región conurbada de Florianópolis, diferente de aquellos estudios clásicos de clima urbano, pues comprende sedes de cuatro municipios con características urbanas, en una región cuya ocupación es nucleada, conforme admite el propio Instituto de Pla- neamiento Urbano de Florianópolis (IPUF) en su modelo de urbanización de la ciudad. Solo en el sector central de la región ocurre una urbanización continua, dificultando la delimitación intermunicipal, configurando la conurbación.

Los resultados alcanzados evidencian la relación entre la ocupación urbana y la isla de calor, con sus variaciones espaciales. Entretanto, la compartimentalización morfológica de la región estudiada, determinando diferentes disposiciones para las vertientes en relación a la insolación y a la circulación de los vientos, ya sea aislando, o exponiendo determinadas áreas, dificulta, muchas veces, la determinación del peso de esta influencia. Es importante resaltar aun la fuerte influencia del mar que representó el 65\% del área delimitada para la realización del mapa de Uso de la tierra.

La conservación térmica observada en los mapas de gradientes de temperatura demuestra la necesidad urgente de un replaneamiento de los dos usos, creando obstáculos a la construcción de torres, aumentando las separaciones entre construcciones, disminuyendo la densificación e incentivando, a través de un servicio barato y eficiente, el transporte colectivo. Además de eso, debería ser mantenida la vegetación arbórea remanente en los intersticios de la mancha urbana y fomentada la implementación de otros espacios de este tipo, cambiando el concepto de las denominadas áreas verdes de descanso y recreación que se limitan a superficies disponibles para la práctica de deportes. Estas medidas son fundamentales para mejorar el confort térmico $y$, consecuentemente, la calidad de vida de los habitantes.

El actual planeamiento realizado en Florianópolis privilegia la especulación inmobiliaria y las industrias de construcción civil y automovilística en detrimento de la calidad del aire y de la vida. La densificación de las construcciones, la multiplicación de las torres y la sucesión de áreas de rellenos de terreno para la ampliación del sistema viario, en detrimento de la preservación de ambientes como ensenadas, manglares y campos de dunas, tienden a perjudicar la actividad turística que es fetichizada por los 
gestores públicos y por los sectores privados. Se percibe que, por la motivación empresarial actual, la tendencia es a transferir, definitivamente, el modelo de ocupación urbana densa para los balnearios y periferias de la región, intensificando las islas de calor ya esbozadas en las áreas urbanas menos densas y consolidando un "archipiélago" de calor.

No es posible todavía evaluar las consecuencias de esta opción para cada uno de los mesoclimas identificados en la región. Pocas estaciones fueron establecidas en las zonas Sede y São José, donde ya se puede comprobar un significativo comprometimiento del campo térmico por los factores urbanos, resaltando que la cuestión ambiental local es muy compleja. Estas constataciones remiten a la necesaria continuidad de los estudios de este tipo, para lo cual se debe profundizar el detalle con una mayor densidad de estaciones y de la sofisticación de los aparatos utilizados para los registros.

\section{Agradecimientos}

A los estudiantes de las carreras de Geografía e Ingeniería de las universidades Federal y Estadual de Santa Catarina y a los demás auxiliares voluntarios de los trabajos de campo.

Al apoyo técnico y colaboración de los profesores Joel Pellerin y Luiz Antônio Paulino y del geógrafo José Henrique Vilela, del Laboratorio de Geoprocesamiento del Departamento de Geociencias de la UFSC, por la realización del geoprocesamiento y mapeo de los datos.

\section{Referencias bibliográficas}

CLIMANÁLISE. Aspectos da climatologia dinâmica no Brasil. Boletim de monitoramento e análise climática, 1986, número especial, 1986.

DANNI, I. Aspectos têmporo-espaciais da temperatura e umidade relativa de Porto Alegre en janeiro de 1982: contribuição ao estudo do clima urbano. Dissertação de Mestrado. São Paulo: Departamento de Geografia, Faculdade de Filosofía, Letras e
Ciências Humanas, Universidade de São Paulo, Brasil, 1987.

GOVERNO DO ESTADO DE SANTA CATARINA. Plano básico de desenvolvimento ecológico-econômico (PBDEE). Florianópolis: Secretaria de Estado do Desenvovimento Urbano e Meio Ambiente (SDM) e Associação dos Municípios da Grande Florianópolis (GRANFPOLIS), 1996.

HERRMANN, M. Problemas geoambientais na faixa central do litoral catarinense. Tese de Doctorado. São Paulo: Departamento de Geografia, Faculdade de Filosofía, Letras e Ciências Humanas, Universidade de São Paulo, Brasil, 1999.

INSTITUTO BRASILEIRO DE GEOGRAFIA E ESTATISTICA (IBGE). Sinopse preliminar do censo demográfico 2000. Santa Catarina (Recenseamento Geral do Brasil, 2000). Rio de Janeiro: IBGE, 2000.

INSTITUTO DE PLANEJAMENTO URBANO DE FLORIANÓPOLIS (IPUF). Modelo de ocupação. Florianópolis: IPUF, 1985. Disponible en Internet: http:/ www.ipuf.sc.gov.br/pages/planejamento/ gpl_modelo.asp

INSTITUTO NACIONAL DE METEOROLOGIA (INMET). Condições do temp registradas nas capitais do país. Meteorologia sinótica INMET. Brasília: INMET, 1999-2000. Disponible en Internet: http//www.inmet.gov.br/port/sinótica/ param.htm

LOMBARDO, M. Ilha de calor nas metrópoles - o exemplo de São Paulo. São Paulo: HUCITEC, 1985.

MENDONÇA, M. A dinâmica têmporoespacial do clima subtropical na região conurbada de Florianópolis/SC. Tese de Doutorado, Departamento de Geografia, Faculdade de Filosofia Letras e Ciências Humanas, Universidade São Paulo, Brasil, 2002.

MENDONÇA, M. \& LOMBARDO, M. A dinâmica têmporo-espacial do clima subtropical na região conurbada de 
Florianópolis/SC. In: SBCG. Contribuições científico-técnicas do V SBCG. Curitiba: V Simpósio Brasileiro De Climatologia Geográfica (SBCG), 2002, p. 450-462.

MENDONÇA, M.; PAULINO, L.; PELLERIN, J. \& VILELA, J. Mapeamento do campo térmico no Centro de FlorianópolisPrimeiras etapas de uma proposta metodológica. In: SBGFA. Anais do XII SBGFA-Simpósio Brasileiro de Geografia Física Aplicada. Natal: XII Simpósio brasileiro de geografia física aplicada, Departamento de Geografia da Universidade Federal do Rio Grande do Norte, 2007, p. 1-5.

MONTEIRO, C. Geografia regional do Brasil - região sul (Tomo 1, cap. III). Rio de Janeiro: IBGE, Série Biblioteca Brasileira, 1963.

MONTEIRO, C. A Frente Polar Atlântica e as chuvas de inverno na fachada Sul Oriental do Brasil (Contribuição metodológica à análise rítmica dos tipos de tempo no Brasil). São Paulo: Instituto de Geografia, Universidade São Paulo, Ilustr. São Paulo, Série Teses e Monografias $N^{0} 1$, 1969.

MONTEIRO, C. Por un suporte teórico e prático para estimular estudos geográficos do clima urbano no Brasil. Geosul, 1990a, No 9, p. 7-19.

MONTEIRO, C. Adentrar a cidade e tomar-lhe a temperatura. Geosul, 1990b, No 9, p. 61-79.
MONTEIRO, C. A cidade como processo derivador ambiental e estrutura geradora de un "clima urbano". Geosul, 1990c, № 9, p. 80-114.

MONTEIRO, C. \& SEZERINO, M. O campo térmico da cidade de Florianópolis: primeiros experimentos. Geosul, 1990, No 9, p. 21-60.

MONTEIRO, M. Avaliação das condições atmosféricas de Florianópolis para controle da Qualidade do ar. Graduação en Geografia. Florianópolis: Departamento de Geociências da Universidad Federal de Santa Catarina, Brasil, 1992.

ROSA, R. \& HERRMANN, M. Aerofoto Cruzeiro de Sul. In: GAPLAN. Atlas de Santa Catarina. Rio de Janeiro: GAPLAN, 1986.

SERRA, A. Circulação superior. Revista Brasileira de Geografia, 1954, vol. 15, № 4, p. 517-596.

SERRA, A. \& RATISBONNE, L. Massas de ar na América do Sul. Rio de Janeiro: Serviço de Meteorologia, Ministério da Agricultura, 1942.

SEZERINO, M. \& MONTEIRO, C. O campo térmico na cidade de Florianópolis: primeiros experimentos. Geosul, 1990, Nº 9, p. 29-60.

STRAHLER, A. Geografía física. Barcelona: Omega, 1975. 